\title{
ASPERGILOSE CEREBRAL EM PACIENTE IMUNOCOMPETENTE
}

\author{
Geraldo Pianetti Filho', Enio Roberto Pietra Pedroso², \\ Alexandre Varela Giannetti ${ }^{3}$, Rogério Darwich ${ }^{4}$
}

\begin{abstract}
RESUMO - Descrevemos um caso raro de aspergilose cerebral, como complicação de ciru rgia para aneurisma c e rebral, em paciente imunocompetente, e com sucesso no tratamento. A paciente, de 40 anos, havia sido submetida a craniotomia para clipagem de aneurisma da artéria pericalosa. Após cinco meses, no sitio cirúrgico, surgiram múltiplos abscessos por Aspergillus sp. Foram necessárias duas craniotomias para a retirada da massa fúngica e uso de anfotericina B. Após 14 anos de acompanhamento, a evolução mostra resolução da aspergilose, sem seqüela. $O$ tratamento do abscesso cerebral por Aspergilus exige acompanhamento clínico contínuo, repetidas drenagens cirúrgicas intracranianas e o uso de anfotericina B no tratamento medicamentoso.
\end{abstract}

PALAVRAS-CHAVE: aspergilose, abscesso cerebral, micose oportunística.

\section{Cerebral Aspergillus abscess in immunocompetent patient}

ABSTRACT - We re p ortan unusual case of brain aspergillosis with multiple re cu rrent abscess in a 40 yearsold immunocompetent woman, with good therapeutical outcome. The patient presented a subaracnoid hemorhage caused by a ruptu red pericalosal art e ry aneurysm and was submitted to a craniotomy for aneurysm surg ery. Five months later, she developed multiple Aspergillus cerebral abscess. Two craniotomies and amphotericin B became necessary during treatment. Fourteen years later, she is asymptomatic. Treatment of brain aspergillosis abscess implied the combination of both surgical and drug therapy with amphotericin B.

KEY WORDS: aspergillosis, brain abscess, opportunistic mycosis.

A aspergilose re presenta um espectro patológico determinado por espécies do gênero Aspergillus (fumigatus, flavus, niger, terreus, sudowi, us tus, versicolor, amstelodami, oryzae, candidus, res trictus, nidulans), que são os fungos mais comuns do planeta, presentes no solo, adubo, feno, cereais, vegetais e até em ambiente hospitalar. Penetram no organismo humano por várias vias, especialmente, inalatória e se alojam inicialmente, nos seios para-nasais $^{1-3}$ e a seguir invade as vias aéreas inferiores. O pulmão é o órgão predominantemente envolvido. Os neutrófilos, macrófagos e monócitos são fundamentais para a sua destruição. O fungo, se for capaz de ultrapassar as defesas humanas, é angiotrópico, promovendo destruição vascular com infarto, necrose, trombose e hemorragia texturais. $\mathrm{O}$ acometimento de pessoas imunocompetentes caracteriza-se, principalmente, por reação de hipersensibilidade dirigida ao aparelho respiratório e envolvimento saprofítico de cavidades pulmonares (caverna). A sua capacidade de invasão de órgãos e tecidos (invasão pulmonar, abscesso ce rebral, infecção disseminada) associa-se, usualmente, com imunossupressão de várias etiologias. $\mathrm{O}$ acometimento do sistema nervoso central (SNC) é muito grave, com $75 \%$ a $100 \%$ de morte, apesar do tratamento intensivo com anfotericina B (an$f B)^{4,5}$. O diagnóstico é difícil, freqüentemente re alizado à necropsia ${ }^{4-9}$. A preocupação com a aspergilose cerebral é assunto atual devido ao aumento de sua incidência nos últimos anos, em decorrência, principalmente, do maior uso de imunodepressores $^{2,6,10-12}$, da disseminação do vírus da imunodeficiência humana (VIH) e da síndrome da imunodeficiência adquirida (SIDA)7. A invasão do SNC por Aspergillus sp. pode ocorrer pela via hematogênica, como manifestação da disseminação sistêmica, ou por contigüidade ${ }^{13}$, pelas estruturas contaminadas, principalmente pelos seios para-nasais e pela mastóide, após traumatismo crânio-encefálico,

Se niços de Neurociru rgia e Clínica Médica do Hospital das Clínicas (HC) da Universidade Federal de Minas Gerais (UFMG), Belo Horizonte MG, Brasil: ${ }^{1}$ Professor Adjunto do Departamento de Cirurgia da Faculdade de Medicina da UFMG (FM), Neurocirurgião do HC; ${ }^{2}$ Professor Titular do Departamento de Clínica Médica da FM; ${ }^{3}$ Neurocirurgião do HC; ${ }^{4}$ Neurologista.

Recebido 15 Outubro 2004, recebido na forma final 10 Setembro 2005. Aceito 12 Setembro 2005.

Dr. Geraldo Pianetti Filho - Rua dos Aimorés 2480/902 - 30140-072 Belo Horizonte MG - Brasil. E-mail: pianettigpf@aol.com.br 


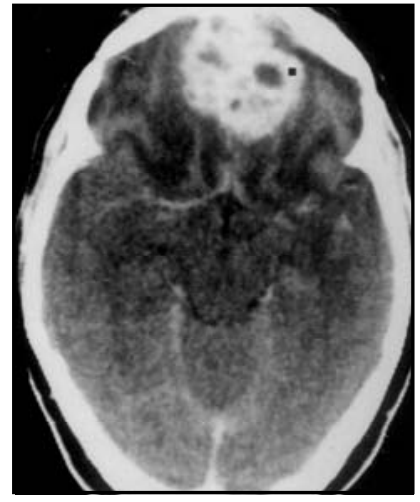

Fig 1. TC pré tratamento, mos trando o abscesso.

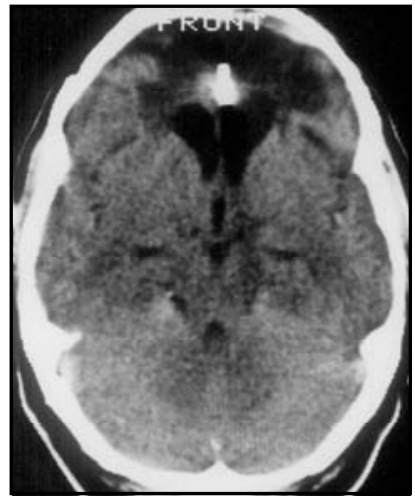

Fig 2. TC realizada após o trata mento. Observa-se imagem do clip metálico usado na cirurgia do aneurisma.

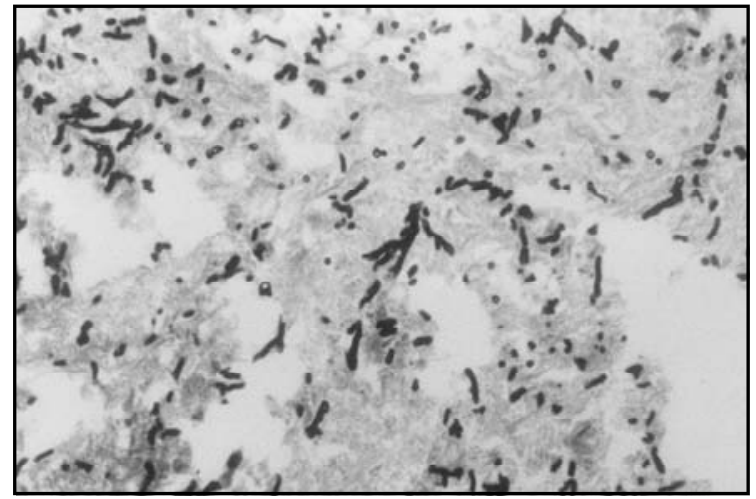

Fig 3. Imagem panorâmica da lâmina histológica, mostran do o fungo. por punções lombares repetidas para tratamento intratecal com penicilina ${ }^{2}$ e ainda após manipulação neurocirúrgica ${ }^{10,14-16}$.

O primeiro caso de Aspergillus no SNC após uma neurocirurgia foi descrito em $1973^{17}$. Este trabalho descreve caso semelhante, em que uma paciente evoluiu com abscessos frontais por $A s p$ e rgillus $s p$, após o tratamento cirúrgico de um aneurisma intracraniano. A diferença deste caso está na higidez prévia à neurocirurgia, da paciente objeto deste relato, em que, apesar da reserva do prognóstico, obtevese sucesso terapêutico com a combinação de cirurgia de drenagem e uso de anfB. Parece ser o primeiro caso descrito no Brasil de sucesso terapêutico na aspergilose do SNC após neurocirurgia.

\section{CASO}

Mulher de 40 anos, hígida até dezembro de 1989, quando apresentou cefaléia fronto-occipital de início súbito, seguida de fraqueza muscular nos membros à direita e perda momentânea da consciência. Foi atendida em serviço de urgência. Apresentava-se consciente, normotensa, com sinais de irritação meníngea e hemiparesia com predomínio crural à direita. A tomografia computadorizada do crânio (TC) revelou sangue na região frontal esquerda, o estudo angiográfico do sistema ca rotideano mostrou dilatação aneurismática na artéria pericalosa esquerda, na emergência da artéria calosa marginal. No décimo quarto dia após o sangramento, quando já estava recuperada da hemiparesia, foi submetida a craniotomia bifrontal com clipagem do aneurisma. O pós-operatório transcorreu sem anormalidades e recebeu alta em boas condições, sem lesões neurológicas. Cinco meses após voltou a apresentar, na região frontal, cefaléia intensa, e outros sinais inflamatórios. Nova TC revelou abscesso intimamente relacionado com o seio frontal, que havia sido aberto durante a cirurgia anteriomente realizada. Foi submetida a punção aspi- rativa transcutânea, com obtenção de secreção purulenta, que foi drenada. $O$ exame direto, em lâmina, deste material, revelou hifas que a cultura identificou As pergillus sp. (Fig 1). Foi medicada com fluconazol durante 40 dias com melhora da sintomatologia.

Manteve-se assintomática até cinco meses após a interrupção do anti-fúngico quando, de novo, voltou a ter cefaléia e edema frontais. Estava anósmica, com anemia n o rmocrômica e velocidade de hemossedimentação elevada, leucócitos e plaquetas numericamente normais. A TC mostrou abscessos frontais (Fig 2). Foi submetida a craniotomia com drenagem dos abscessos, retirada de p rocesso inflamatório e cicatricial aderido à dura-mater, cu retagem e limpeza da cavidade do seio frontal. O estado anátomo-patológico do material curetado evidenciou presença deAspergillus sp (Fig 3). A sorologia para o VIH era negativa. Foi iniciado o tratamento com anfB que provocou vômitos incoercíveis, o que exigiu sua suspensão. Voltou a receber fluconazol por 30 dias, obtendo alta, sem queixas, em boas condições clínicas. Em maio de 1991, 18 meses após o tratamento inicial do aneuris$\mathrm{ma}$, re a pa receu a mesma sintomatologia de cefaléia e edema frontal, e a TC revelou, novamente, abscessos frontais múltiplos. Foi submetida a craniotomia com drenagem dos abscessos e administrada anfB, inicialmente em regime hospitalar e depois ambulatorial.

Após as primeiras doses de anfB surgiram convulsões generalizadas, que foram tratadas com fenitoína. No quarto mês de tratamento apresentou acidemia e hipopotassemia grave (1 $\mathrm{mEq} / \mathrm{L})$, sem elevação das escórias nitrogenadas, que foram corrigidas, sem suspensão da anfB. A dose acumulativa total da anfB chegou a 4800 $\mathrm{mg}$, em 10 meses de tratamento, interrompida em março de 1992, com recuperação plena do estado geral e da anemia, tendo desenvolvido discreta euforia. As TC realizadas até dois anos após o tratamento não mostraram sinais de recidiva. $O$ último controle, feito 14 anos após a primeira neurocirurgia, continuou mostrando paciente assintomática, sem interco rrências, sendo considerada curada. 


\section{DISCUSSÃO}

Trata-se, este caso, do primeiro citado na literatura brasileira, e dos primeiros na literatura mundial, em que o envolvimento do SNC pelo Aspergillus sp., após craniotomia, evoluiu com plena recuperação do paciente.

A aspergilose do SNC possui propensão para localizar-se nas cavidades supratentoriais ${ }^{14}$, e elevada mortalidade apesar do uso de anfB ${ }^{1,4,6,9}$. Provêm geralmente de disseminação hematogênica ou por contigüidade a partir das estruturas aeradas da face e crânio. A disseminação pode ocorrer desde a meninge, causada por hifas, abscessos produzidos por pseudo-hifas e infarto cerebral decorrente de ramos das hifas que obstruem vasos grandes e intermediários provocando trombose arterial cerebral ${ }^{16,17}$. A invasão vascular secundária pode resultar em aneurisma micótico e hemorragia intraparenquimatosa ${ }^{16,17}$. As hifas podem bloquear os vasos sangüíneos intracerebrais, promover infarto, comumente hemorrágico e estéril, que pode evoluir para abscesso sépti$\mathrm{CO}^{2,3,6,16-18}$, e promover reação inflamatória mista e necrose $^{6,16}$, vasculite e aneurisma micótico ${ }^{3,16,17}$ quando o fungo causa erosão através da parede do vaso para o interior do parênquima cerebral isquêmico.

A infecção por contigüidade, em pacientes imunossuprimidos, geralmente se associa ao acometimento dos seios paranasais e relaciona-se com condições de intensa invasividade fúngica. OAspergillus sp. pode penetrar a órbita e promover proptose, quemose e necrose cutânea. A extensão direta da órbita para o lobo frontal pode rapidamente provocar trombose do seio cavemoso. A destruição óssea, infiltração retro-orbitária e formação de abscessos podem ser evidentes ${ }^{12}$. Os vasos cerebrais podem ficar ocluídos pelo Aspergillus e com infarto cerebral. O abscesso cerebral apresenta necrose localizada com numerosas hifas ao redor de tecido de granulação denso. As pessoas imunocompetentes, embora raramente, podem ter quadros semelhantes com granulomas e vasculites. A meningite devida ao Aspergillus é também rara e associa-se com o uso de drogas ilícitas ou cirurgia trans-esfenoidal ${ }^{19}$.

As imagens à $\mathrm{TC}^{2,3,6}$ revelam lesões de baixa densidade, com pequeno ou nenhum efeito de massa e sem realce com contraste. A presença de imagem anelar ou nodular consistente com o diagnóstico de abscesso ou granuloma ${ }^{3}$ indica que as defesas sistêmicas do hospedeirosão hábeis em isolar e encapsular o agente agre ssor. A parede do anel é freqüentemente fina e mais irregular do que a do abscesso piogênico. A neurocirurgia também pode ser fonte de infecção.

Existem poucas descrições de pacientes com aspergilose cerebral e que sobreviveram após serem submetidos a intervenção cirúrgica e terapia anti-fúngica ${ }^{6,12,16,18}$. A infecção fúngica que se apresenta como massa deve ser tratada pela ressecção cirúrgica agressiva. O paciente que apresenta recorrência ${ }^{7}$ pode se beneficiar de aplicação da anfB intralesional ${ }^{20}$.

Os fatores que predispõem a aspergilose invasiva ${ }^{2,16}$ associam-se com o tratamento com altas doses de glicocorticóides (como nos linfomas, nas doenças autoimunes, nos transplantes de órgãos), o hipercorticismo suprarenal (síndrome de Cushing), a granulocitopenia intensa e persistente (quimioterapia para a leucemia, linfoma e transplante de medula óssea), a doença granulomatosa crônica, a SIDA. A virulência do agente é também fator de grande importância para a patogenicidade da aspergilose. A re corrência da aspergilose pulmonar é observada em $50 \%$ dos pacientes que receberam ciclos intensivos de quimioterapia para o câncer. Esta mesma propensão é encontrada após cirurgia do SNC.

O diagnóstico diferencial da imunodeficiência em paciente com infecções recorrentes é complexo por envolver características semelhantes a patologias que envolvem anormalidades dos neutrófilos, linfócitos, monócitos, anticorpos e complemento. A maioria dos pacientes não apresenta defeito identificável dos fagócitos ou imunodeficiência. Em geral, deve-se efetuar uma avaliação no decorrer de um ano. Neste período, para caracterizar alguma imunodeficiência, os pacientes devem apresentar, pelo menos, uma das seguintes alterações clínicas: 1. mais de duas infecções bacterianas sistêmicas (septicemia, meningite, osteomielite), 2. três infecções respiratórias graves (pneumonia, sinusite), ou três bacterianas (celulite, otite média com drenagem, linfadenite); 3. infecção em local pouco habitual (abscesso hepático ou cerebral); 4. infecções por patógenos pouco comuns (pneumonia por Aspergillus, candidíase disseminada ou infecção por Serratia marcescens,por espécies de Nocar dia e de Berkoldaria cepacia) e; 5 . infecções muito graves ${ }^{21}$.

Os pacientes com defeitos da adesão e da motilidade dos neutrófilos geralmente desenvolvem microabscessos cutâneos e formações granulomatosas associados a patógenos comuns como S. aureus, S. marcescens, Pseudo monas, Aspergillus ou apresentam lesões mucosas pela C. albicans. Há escassez de neutrófilos no local da inflamação. Ocorrem também linfadenopatia recorrente, abscessos hepáticos bacterianos, pneumonia, linfadenite, infecções cutâneas. Podem evoluir com anemia, dermatite purulenta crônica, hepatoespleno e linfadenomegalias, doença pulmonar restritiva, gengivite, hidronefrose, estenose gastrintestinal, osteomielite em múltiplos locais ou nos ossos pequenos das mãos e dos pés. Há história familiar de infecções recorrentes ou por micorg anismos catalase-positivos. A sintomatologia inicia desde os primórdios da vida até o início da vida adulta. Na deficiência de mieloperoxidase o principal problema consiste no aumento das infecções pela Candida em alguns pacientes portadores de diabetes mellitus. Na deficiência de adesão leucocitária ocorre leucocitose significativa, separação retardada do cordão umbelical, onfalite, gengivite, infecções cutâneas e otite média recorrentes, pneumonite, peritonite, abscessos anais e deficiências na cicatrização das feridas. Os neutrófilos podem também apresentar redução da adesividade e da quimiotaxia após exposição a numerosos fármacos, entre os mais 
comuns estão os corticóides e a epinefrina, e manifestase por acentuada neutrofilia, infecções recorrentes dos tecidos moles, demora na cicatrização das feridas e acentuada redução da formação de pus. As manifestações começam no período neonatal e a sobrevivência não ultrapassa dois anos de idade. Na síndrome de Chédiak-Higashi há neutropenia, febre alta, hepatoesplenomegalia sem septicemia bacteriana. Na febre familiar do mediterrâneo as infecções recidivantes ocorrem uma a duas vezes por mês ou mesmo por semana com serosites, artrites e até amiloidose renal.

As deficiências na função dos linfócitos T (LT) geralmente manifestam-se por infecções oportunísticas. Há ausência de corpúsculos de Howell-Jolly; as contagens n o rmais de plaquetas, de neutrófilos e de linfócitos, no sangue periférico, ajudam a excluir as síndromes de asplenia, de Wiskott-Aldrich, as neutropenias congênitas e adquiridas, os defeitos graves da quimiotaxia, a possibilidade de defeito grave dos $\mathrm{LT}^{22}$.

$\mathrm{Na}$ agamaglobulinemia ligada ao $\mathrm{X}$ aparecem infecções por microrganismos piogênicos extracelulares agressivos como pneumococos, estreptocoos e espécies de Haemophilus, entre seis a nove meses após o nascimento. As infecções mais comuns são sinusite, pneumonite, otite, artrite séptica, meningite, septicemia. Em geral não ocorrem infecções fúngicas crônicas. Na imunodeficiência variável comum as infecções recorrentes, ocorrem muitos anos após o nascimento, associa-se com síndrome espru-símile, com timoma, alopécia em áreas, anemia hemolítica, atrofia gástrica, acloridria, anemia perniciosa. A deficiência seletiva de IgA relaciona-se com infecções p redominantemente nos aparelhos respiratório e gastrintestinal e excessiva susceptibilidade às infecções virais. $\mathrm{Na}$ imunodeficiência com níveis elevados de IgM o habitual é a sintomatologia desde a lactância, com infecções piogênicas recorrentes, caracterizadas por otite, sinusite, pneumonite e amigdalite. Na doença linfoproliferativa ligada ao $\mathrm{X}$ ocorre resposta imune inadequada à infecção pelo vírus Epstein-Barr. As pessoas são sadias até apresentarem mononucleose infecciosa, e manifestase geralmente antes dos cinco anos de idade. Na hiperimunoglobulina E há dermatite crônica e infecções sinopulmonares recorrentes.

As deficiências hereditárias do complemento estão associadas ao lupus eritematoso sistêmico, glomeru n efrites, vasculites e infecções piogênicas recorrentes e disseminadas relacionadas com a Neisseria, o angioedema he reditário (lesões cutâneas, meningite meningocóccica, crise episódicas de edema circunscrito não-pruriginoso da pele ou da mucosa das vias aéreas ou do trato gastrintestinal).

Na hipoplasia tímica (síndrome de DiGeorge) ocorrem anomalias dos grandes vasos (arco aórtico direito), a tresia do esôfago, úvula bífida, cardiopatia congênita, hipoplasia mandibular. Nos distúrbios de imunodeficiência combinada grave (IDCG) surge no lactente otite, pnemonite, septicemia, diarréia, infecções cutâneas. As infecções persistentes são relacionadas a microrganismos oportunistas como $C$. albicans, $P$. carinii e vírus da varicela, do sarampo, parainfluenza 3 e citomegálico. As suas variantes (recessiva ligada ao $\mathrm{X}$, autossômica recessiva, deficiências enzimáticas por adenosina desaminase, Janus quinasse 3 , RAG-1 ou RAG-2, IL-7Ralfa, ou com leucopenia) são todas de evolução semelhantes a IDCG. Outros distúrbios da imunodeficiência combinada decorrentes de deficiências de purina nucleosídeo fosforilase, das mutações da cadeia alfa do receptor da interleucina 2, da ativação deficiente dos LT, da expressão defeituosa dos antígenos do complexo de histocompatibilidade principal, da imunodeficiência com trombocitopenia e eczema (síndrome de Wiskott-Alddrich), da ataxiatelangiectasia (ataxia cerebelar com telangiectasias oculocutâneas, doença sinopulmonar crônica, elevada incidência de processos malignos), ou a hipoplasia da cartilagem-cabelo (nanismo com membros curtos, infecções freqüentes e graves), a função dos LT está diminuída, porém não ausente. Os pacientes apresentam, desde recém-nascidos, infecções pulmonares, cutâneas, urinárias, intestinais recorrentes ou crônicas, septicemia por Gram negativos, ou varicela grave.

As mutações de interferon gama R1 e da interleucina $12 \mathrm{R}$ beta 1 associam-se com infecções disseminadas pelo BCG. Nas deficiências primárias do sistema do complemento há susceptibilidade aumentada às infecções por microrganismos Gram positivos encapsulados, por meningococos ou gonococos.

A aspergilose invasiva ocorre especialmente em pacientes imunossuprimidos, tendo como fatores predisponentes altas doses de esteróides sistêmicos, a granulocitopemia intensa, a doença granulomatosa. O envolvimento saprofítico do trato respiratório decorre da contaminação de cavidades pré-existentes ou bronquiectasias que se desenvolvem na tuberculose ou sarcoidose. As crianças com fibrose cística e broquiectasia podem também apresentar condições de crescimento saprofítico para as espécies de Aspergillus,que se desenvolvem no c e rume e restos descamados no conduto auditivo externo. Na imunodeficiência intensa pode haver progressão para a mastóide e o SNC.

A apresentação mais freqüente da aspergilose em pacientes sem imunodeficiência é a forma de alveolite alérgica extrínseca que surge em repetidas exposições ao fungo em trabalhadores não-atópicos (pulmão dos fazendeiros). Pode também provocar sinusite alérgica (em paciente atópico com polipose nasal) e aspergilose a lé rgica broncopulmonar. A sinusite invasiva por aspergilose ocorre raramente em hospedeiros normais. Devese evitar a lesão encefálica e a hipertensão intracraniana, em paciente com aspergilose, pelo risco de favorecer a invasão cerebral pelo Aspergillus.

A patogenicidade das espécies de Aspergillus, embora dependa primariamente da imunossupressão, decorre também de fatores de virulência, que sob certas condições in vitro, algumas cepas sintetizam proteases tais como elastase, implicada na invasão do tecido pulmonar. Algumas cepas de A. fumigatus eflavus produ- 
zem um inibidor do complemento que podem aumentar a patogenicidade pela subversão da geração de opsoninas e fatores quimiotáticos. O A. fumigatus p roduz a gliotoxina que possui, in vitro, atividade imunossupressora e antifagocítica. A ação destas toxinas sobre o ser humano é ainda desconhecida.

O diagnóstico da aspergilose do SNC é difícil em deconência da sintomatologia inespecífica6 e ausência de síndrome febril ${ }^{13}$. O acometimento pulmonar nem se $\mathrm{m}$ preestá presente. $\mathrm{O}$ exame de líquor revela alterações sutis, com pequena elevação das proteínas. O Asper gillus sp, é raramente cultivado a partir do líquor ${ }^{11,13}$. Por estes motivos e pela gravidade do prognóstico, o diagnóstico por meio de técnica intervencionista deve ser avaliado.

O caso clínico aqui descrito apresenta características singulares que excluem os vários fatores que promovem imunodeficiência, ainda reforçados pelo longo tempo de acompanhamento, 14 anos, com total ausência de sintomas após o tratamento da aspergilose.

Apesar da presenação da imunidade nesta paciente, as recidivas foram muitas, o risco de morte elevado; ent retanto, apesar das adversidades, a persistência da terapêutica combinada, cirúrgica e clínica, conseguiram a sua recuperação completa. $O$ tratamento clínico é recomendado até duas semanas após a recuperação da medula óssea quando há neutropenia ${ }^{23}$. O contato direto da anfB com o SNC pode provocar cefaléia, delírios, vômitos, parestesias, convulsões, aracnoidite, radiculopatias, mielopatias, meninigte, plegias. Neste caso a convulsão associada ao teste inicial com a anfB, impediu o seu uso intratecal. As formulações lipossomais, apesar de dispendiosas, não estavam disponíveis na época em que a paciente foi tratada.

A excisão cirúrgica tem sido de sucesso para tratar a aspergilose invasiva do cérebro ${ }^{24}$, e dos seios paranasais ${ }^{25,26}$, assim como a colonização não-invasiva dos seios da face ${ }^{27}$. A anfB é a droga de escolha para a aspergilose invasiva. A eficácia é melhor quando o diagnóstico é precoce, a imunossupressão não é intensa e a sua dose é rapidamente administrada para atingir seus níveis terapêuticos ${ }^{28-31}$. O sucesso terapêutico evidencia que a aspergilose encefálica deve ser tratada com perseverança, de forma a realizar tantas cirurgias quantas forem necessárias para drenar a lesão fúngica, associada ao tratamento medicamentoso com a anfB.

Em conclusão, a aspergilose do SNC em paciente imunodeprimido possui prognóstico reservado, a morte advém rapidamente, e é também grave no paciente imunocompetente. A terapêutica deve ser a mais precoce, tentando a combinação com a ressecção cirúrgica da massa tantas vezes quantas forem necessárias associadas ao uso de anfotericina B.

\section{REFERÊNCIAS}

1. Cox J, Murtagh FR, Wilfog A, Brener J. Cerebral aspergillosis MR imaging and histopathologic correlation. AJNR 1992;13:1489-1492.

2. Enzmann DR, Brant-Zawadzki M, Britt RH. CT of central nervous system infections in immunocompromised patient. AJNR 1980;1:1239-1243.
3. Grossman RI, Davis KR, Taveras JM, Beal MF, O'Carrol CP. Computed tomography in intracranial aspergillosis. J Comput Assist Tomogr 1981; 5:646-650.

4. Denning DW, Stevens DA. Antifungal and surgical treatment of invasive aspergillosis: review of 2121 published cases. Rev Infect Dis 1990; 12:1147-1201.

5. Young RC, Bennett JE, Vogel CL, Carbone PP, DeVita VT. Aspergillosis: the spectrum of the disease in 98 patients. Medicine 1970;49:147-174.

6. Beal MF, O'Carrol P, Kleinman GM, Grossman RI. Aspergillosis of the nervous system.Neurology 1982;32:473-479.

7. Darras-Joly C, Veber B, Bedos JP, Gachot B, Regnier B, Wolff M. Nosocomial cerebral aspergillosis: a report of three cases. Scand J Infect Dis 1996;28:317-319.

8. Mathews MS, Chandy MJ. Carebellar granuloma caused by Aspergillus fumigatus: first report from India. Trans R Soc Trop Med Hyg 1995; 89:83-84.

9. Wash TJ, Hier DB, Caplan LR. Aspergillosis of the sentral nervous system: clinicopathological analysis of 17 patients. Ann Neurol 1985;18:574-582.

10. Camarata PI, Dunn DI, Farney AC, Parker RG, Seljeskog EI. Continual intracavitary administration of amphoterecin $\mathrm{B}$ as an adjunct in the treatment of Aspergillus brain abscess:case report and reviw of the literature. Neurosurgery 1992;31:575-579.

11. Cohen J. Clinical manifestations and managment of aspergillosis in the compromised patient. In Warnock DW, Richardson MD. Fungal infection in the compromised patient. London: John Wiley \& Sons, 1991:117-151.

12. Epstein NE, Hollingsworth R, Black K, Framer P. Fungal brain abscess (aspergillosis/mucormycosis) in two immunosuppressed patients. Surg Neurol 1991;35:286-289.

13. Lyons RW, Andriole VT. Fungal infection of CNS. Neurol Clin 1986; 4:159-170.

14. Bjorkholm B, Elgefors B. Cerebellar aspergilloma. Scand Infect Dis 1986;18:375-378.

15. Maschke M, Dietrich U, Prumbaum M, Kastrup O, Turowski B, Schaefer UW. Opportunistic CNS infections after bone marrow transplantation. Bone Marrow Transplant 1999;23:1167-1176.

16. Miaux Y, Ribaund P, Williams M, et al. MR of cerebral aspergillosis in patients who have had a bone marrow transplantation. AJNR 1995; 16:555-562.

17. Sharma RR, Gurusinghr NT, Lynch PG. Cerebral infarction due to aspergillus arterits following glioma surgery. Br J Neurosurg 1992;6:485-490.

18. Aschdown BC, Tien RD, Felsberg GJ. Aspergilosis of the brain and paranasal sinuses in imminocompromised patients: CT and MRI imaging findings. Am J Roentgenol 1992;162:155-159.

19. Feely M, Steiberg M. Aspergillus infection complicating transphenoidal yttrium-90 pituitary implant. J Neurosurg 1977;46:530-533.

20. Erdogan E, Beyzadeoglu M, Arpaci F, Celasun B. Cerebellar aspergillosis: case report and literature review. Neurosurgery 2002;50:874-877.

21. WHO Scientific Group. Primary immunodeficiency diseases. Clin Exp Immunol 1997;99:1-24.

22. Buckley RH, Schiff RI, Schiff SF, et al. Human severe combined immunodeficiency (SCID): genetic, phenotypie and functional diversity in 108 infants. J Pediatr 1997;130:378-387.

23. Moraes EM. Micoses profundas. In Rocha MOC, Pedroso ERP, Marques LGM, Silva AO. Terapêutica clínica, Rio de Janeiro: Guanabara Koogan, 1993;1121-1142.

24. Venugopal PV, Venugopal TV, Thiruneelakantan D, et al. Cerebral aspergillosis. report of two cases. Sabouraudia 1977;15:255-258.

25. Green WR, Font RL, Zimmerman LE. Aspergilosis of the orbit: report of ten cases and review of the literature. Arch Ophthalmol 1969;82:302-306.

26. Sandison AT, Gentles JC, Davidson CA, et al. Aspergilloma of paranasal sinuses and orbit in northern sudaneses. Sabouraudia 1967;6:57.

27. Stevens MH. Aspergillosis of the frontal sinus. Arch Otolaryngol 1978; 104:153-155.

28. Aisner J, Schimpff SC, Wiernik PH. Treatment of invasive aspergillosis: relation of early diagnosis and treatment to response. Ann Intern Med 1977;86:539-543.

29. Pennington JE. Successful treatment of Aspergillus pneumonia in hematologic neoplasia. N Engl J Med 1976;308:626-630.

30. Denning DW, Stevens DA. Antifungal and surgical treatment of invasive aspergillosis: review of 2121 published cases. Rev Infect Dis 1990; 12:1147-1201.

31. Levitz SM. Aspergillosis. Infect Dis Clin N Am, 1990;9:1-18. 